Service social

\title{
L'organisation communautaire et les mutations dans les services sociaux au Québec, 1961 - 1991. La marge et le mouvement comme lieux de l'identité
}

\section{Gérald Doré}

Volume 41, numéro 2, 1992

Bilan des réformes

URI : https://id.erudit.org/iderudit/706573ar

DOI : https://doi.org/10.7202/706573ar

Aller au sommaire du numéro

Éditeur(s)

École de service social de l'Université Laval

ISSN

1708-1734 (numérique)

Découvrir la revue

Citer cet article

Doré, G. (1992). L'organisation communautaire et les mutations dans les services sociaux au Québec, 1961 - 1991. La marge et le mouvement comme lieux de l’identité. Service social, 41(2), 131-162.

https://doi.org/10.7202/706573ar
Résumé de l'article

L'auteur traite de l'émergence et de l'évolution de l'organisation communautaire, dans le Québec francophone, dans son rapport aux mutations dans les services sociaux. Il cherche d'abord à la saisir dans l'expérience fondatrice où elle a acquis sa consistance identitaire, soit entre 1961 et 1970, dans les dix années qui ont précédé la première réforme, à la marge à la fois du système de services sociaux privés sous tutelle ecclésiastique et de la profession du service social en conquête de statut. Il suit ensuite son développement, de 1971 à 1990, dans les vingt années donc de la première réforme, en abordant celui-ci sous l'angle du dépassement de la dualité réseau / hors réseau pour la période 1971-1980 et sous l'angle de la persévérance de l'identité face à l'« approche communautaire » et au " néo-bénévolat " dans la période 1981-1990. Il tente en conclusion d'en cerner brièvement les perspectives de ressourcement dans le contexte de la réforme de 1991. 


\section{L'organisation communautaire et les mutations dans les services sociaux au Québec, 1961-1991 \\ La marge et le mouvement comme lieux de l'identité}

Les réformes institutionnelles dans le domaine des services sociaux font partie des contingences avec lesquelles doit composer l'organisation communautaire, en tant que méthode de travail social axée sur le développement de I'action collective des personnes aux prises avec des problèmes sociaux ${ }^{1}$. Ces réformes n'en constituent pas l'essence, cependant. C'est plutôt en effet à la marge des institutions, au point de contact avec le champ social en mouvement, là où se trame l'émergence des solidarités nouvelles et des nouveaux sujets collectifs que l'organisation communautaire se construit et s'affirme dans sa différence spécifique, parmi les autres pratiques sociales qui ont la communauté locale ou la communauté d'intérêts comme terrain d'application. 
Au terme de son processus d'intervention, I'organisation communautaire produit inévitablement de l'institution ; mais si elle s'y résorbe, elle $s^{\prime} y$ perd. Le double mouvement d'identification socio-affective et de conscience critique ${ }^{2}$ dans lequel incubent les solidarités nouvelles débouche, s'il dure, sur des ré-arrangements dans les institutions en place et, éventuellement, de nouveaux arrangements institutionnels. L'organisation communautaire se disperse pour un moment dans les dédales institutionnels au seuil desquels la laisse le mouvement qu'elle a éventuellement contribué à déclencher. Elle ne peut se retrouver telle qu'en elle-même qu'à la condition de se réactiver vers la marge des nouvelles institutions où elle a abouti, là où l'attend son prochain rendez-vous avec le mouvement.

C'est dans le cadre de cette dialectique du mouvement et de l'institution qu'il sera ici question de l'émergence et de l'évolution de l'organisation communautaire dans le Québec francophone, dans son rapport aux mutations dans les services sociaux. Je chercherai d'abord à la saisir dans l'expérience fondatrice où elle a acquis sa consistance identitaire, soit entre 1961 et 1970, dans les dix dernières années qui ont précédé la première réforme, à la marge à la fois du système de services sociaux privés sous tutelle ecclésiastique et de la profession du service social en conquête de statut. Dans le cadre d'analyse qui sous-tend cette réflexion ${ }^{3}$, l'expérience fondatrice est la clé d'interprétation des développements ultérieurs et le lieu de ressourcement de l'identité face aux défis qui se présentent. Nous suivrons ensuite son développement, de 1971 à 1990, dans les vingt années donc de la première réforme, précédée par le Rapport de la Commission d'enquête sur la santé et le bien-être social (Castonguay et Nepveu, 1967-1972) et mise en place dans le cadre de la Loi sur les services de santé et les services sociaux du 24 décembre 1971. Nous tenterons en conclusion d'en cerner brièvement les perspectives de ressourcement dans le contexte de la dernière réforme, préparée par plusieurs documents gouvernementaux, dont le plus fouillé fut le Rapport de la Commission d'enquête sur les services de santé et les services sociaux (Rochon, 1988) ${ }^{4}$, et lancée par la Loi sur les services de santé et les services sociaux et modifiant diverses dispositions législatives, sanctionnée le 4 septembre 1991. L'organisation communautaire est-elle sur le point de se résorber dans une phase de prégnance institutionnelle, inhérente à l'application d'une loi qui fait des « organismes communautaires » des partenaires reconnus de l'État en matière de services sociaux ? Les lieux de réactualisation de sa différence spécifique sont-ils visibles ou prévisibles, à partir de l'analyse des facteurs structurels et culturels qui ont présidé à son émergence et à son développement comme pratique spécifique? 


\section{LE MOMENT FONDATEUR ET LA CONSTRUCTION DE L'IDENTITÉ, À LA MARGE DES SERVICES SOCIAUX PRIVÉS CONFESSIONNELS ET DE LA PROFESSION DU SERVICE SOCIAL EN CONQUÊTE DE STATUT (1961-1970)}

Les événements au cours desquels l'organisation communautaire acquiert son identité comme méthode de travail social sont bien antérieurs à la réforme de 1971. L'organisation communautaire s'affirme en effet comme pratique spécifique en travail social, dès le début des années 60 , dans une aire de mouvement, sur la marge où les vieilles institutions poussent la recherche de leur renouvellement avant de céder graduellement la place à de nouveaux arrangements. Plusieurs repères historiographiques pourraient sans doute être invoqués pour marquer le début de ce processus. Je retiendrai pour ma part un rapport interne du Conseil des œuvres de Montréal, daté de novembre 1961, dans lequel le travailleur social Roger Prud'homme préconise l'implantation dans le quartier Saint-Henri d'un « conseil de quartier », c'est-à-dire une " association bénévole de citoyens et d'organisations d'un secteur donné qui vise à améliorer les conditions de vie de leur milieu et de faire en sorte que les besoins humains, fondamentaux des citoyens puissent être satisfaits » (Prud'homme, $1961: 3$ ).

Ce rapport marque le premier jalon de l'émergence d'une pratique différente de celle désignée jusque-là par le terme « organisation communautaire ». Jusque-là en effet et pour une brève période encore, " organisation communautaire » est un terme servant à nommer l'univers de contenu relatif à la gestion et au développement organisationnel dans les institutions alors en place. En 1962 encore, deux articles publiés dans le même numéro de la revue Service social rendent bien compte de cette acception, dans l'étape achevée de sa formulation, avant qu'elle ne tombe en désuétude pour une longue période. Claude Morin, professeur à l'École de service social de l'Université Laval, assimile l'organisation communautaire à l'organisation des "services privés de bien-être » (Morin, 1962). Pierre Laplante, directeur général du Conseil des œuvres de Montréal, utilise le terme pour désigner l'ensemble des activités de son organisme dont « la préoccupation centrale est de voir à ce que la population ait à sa disposition des services de bien-être adéquats » (Laplante, $1962: 10)$.

Les « services de bien-être » dont il est question renvoient bien évidemment au réseau privé confessionnel institué sous la gouverne de l'Église catholique, à compter des années 30 , sur le modèle anglo-saxon des " community chests » et « community councils ${ }^{5}$ ». Sa structure, lorsqu'elle est complète, comprend pour chaque diocèse : une fédération d'œuvres qui recueille des fonds, un conseil d'œuvres qui développe et coordonne des services et, enfin, des agences qui les dispensent. Après avoir été incorporées 
pour plusieurs d'entre elles sous la Loi des évêques catholiques romains, ces agences sont presque toutes constituées, à la veille de la réforme, en organismes sans but lucratif, en vertu de la troisième partie de la Loi des compagnies. Leur assemblée générale et leur conseil d'administration sont formés de bénévoles, clercs et laïcs dans des proportions variables. Les services qu'elles offrent sont principalement des services sociaux individualisés à la famille et à l'enfance, de plus en plus fournis par des membres de la profession montante du service social. En 1960, tout le Québec est quadrillé par ce réseau qui, malgré son caractère privé et confessionnel, dépend des fonds publics pour au moins $80 \%$ de ses revenus (Perron, $1984: 25$ ).

Le mandat du travailleur social laïc Roger Prud'homme pour l'implantation d'un " conseil de quartier » dans Saint-Henri est à bien des égards représentatif de ce réseau institutionnel, à son apogée, mais sur le point d'aborder son déclin. L'abbé Robert Riendeau, directeur du Conseil des œuvres du diocèse de Montréal, est non seulement clerc, il est lui-même travailleur social et actualise dans sa personne la frontière où l'hégémonie ecclésiastique finissante entre en contact avec la professionnalisation montante dans les services sociaux ${ }^{6}$. II exprime pourtant en même temps une tendance vers la marge de sa profession. Alors que la majorité des membres de celleci est centrée sur une consolidation du statut professionnel autour de la consultation psychosociale individualisée, il en explore la périphérie telle qu'elle se manifeste aux États-Unis. Cette périphérie, c'est l'organisation communautaire, non plus comme terme de substitution à l'administration des services sociaux privés, mais en tant que méthode $d^{\prime}$ intervention participative axée sur le changement social plutôt que personnel. L'abbé Riendeau a fréquenté les expériences d'organisation communautaire de Chicago ${ }^{7}$, rapporte Blondin, et « les a trouvées bonnes» (Blondin, $1970: 299)$. Ces détails historiographiques ne sont évidemment pas rapportés ici pour leur intérêt anecdotique, mais pour mettre concrètement en évidence le lieu social où émerge l'organisation communautaire comme pratique spécifique. Ce lieu est la marge des institutions établies : marge d'initiative laïque à la périphérie d'une hégémonie cléricale en déclin, marge d'orientation collective en bordure d'une profession où l'individualisation des interventions est l'orientation normative dominante.

Aux conditions objectives ou structurelles que je viens d'évoquer correspondent des conditions subjectives ou culturelles. L'intersection des marges institutionnelles est en même temps le point de fusion des tensions éthiques dont elles sont porteuses. La marge de l'institution ecclésiastique est le lieu de réaffirmation de l'option évangélique pour les pauvres et ceux qui souffrent. Au cours des années 50, un prêtre français, fondateur de communautés de sans-abri, les Chiffonniers d'Emmaüs, a été au Québec un porteparole très écouté de cette éthique sociale chrétienne radicale. Son message : 
«Ce qui est nécessaire, au cœur de la société, pour que le pouvoir cesse d'être aveugle et que la souffrance cesse d'être impuissante... c'est la présence de quelques hommes et de quelques femmes, qui, volontaires, décident de s'en aller, non pas pratiquer la bienfaisance, assister ceux qui souffrent, mais se plonger au cœur même de la souffrance de ceux qui souffrent... communautairement, en groupe... » (L'abbé Pierre, 1959 : 48-49) ${ }^{8}$. Parmi les initiatives inspirées de ce message, il y a celle des chantiers étudiants dans les quartiers populaires, initiative à laquelle a participé l'étudiant Michel Blondin, dans le quartier Saint-Henri, avant $d^{\prime} y$ faire son stage en service social avec Roger Prud'homme en 1963 (Blondin, 1970 : 299-300), puis de l'y remplacer en août 1964 (Blondin, $1987: 25)^{9}$.

Bien que le code culturel religieux dans lequel cette tension éthique est exprimée tende à tomber en désuétude dès les années 60 , dans un contexte de sécularisation où le langage des sciences sociales prend le dessus, il ne faut pas sous-estimer son efficacité comme facteur subjectif d'engagement, dans le moment qui fonde l'organisation communautaire dans son identité, non seulement à Montréal, mais dans tous les autres milieux que cette tension idéale a traversés. De nouveaux sujets collectifs émergent dans des populations marginalisées, en conséquence d'une rencontre interclassiste, impulsée par la synthèse $d^{\prime}$ une reviviscence éthique radicale dans la culture traditionnelle ambiante, et d'un emprunt méthodologique, à la marge de la profession du service social, telle qu'elle s'est développée aux États-Unis avec la définition participative de l'organisation communautaire, et notamment les contributions de Ross (1955; Médard, 1969 : 135-145) et d'Alinsky (1969 [1946] ; 1971 ; Médard, 1969 : 159-185).

Blondin reconnaît d'emblée les influences de Ross (Blondin, 1970 : 299) et d'Alinsky (Blondin, 1987 : 26) comme les plus déterminantes de sa méthode, avec celle du « développement communautaire » (Blondin, $1987: 25)^{10}$. Mais le terme de référence qui marquera la conquête d'identité de l'organisation communautaire comme méthode de travail social, jusqu'à la réforme des services sociaux de 1971, n'est directement tributaire d'aucune de ces trois sources d'influence méthodologique. Il est plutôt emprunté, à compter de 1964 (Blondin, 1987 : 25), à l'expérience d' « animation sociale » en cours depuis 1963 dans le Bas-Saint-Laurent et la Gaspésie, sous la responsabilité du Bureau d'aménagement de l'est du Québec (BAEQ, 1966a ; $1966 b$; Morency, 1966) ${ }^{11}$. Cette expérience est alors vécue elle aussi comme un moment fondateur par ses protagonistes, moment fondateur de la planification de l'aménagement du territoire dans la société québécoise en révolution tranquille. Elle est aussi elle-même une pratique à la marge, celle où la technocratie montante concède une place à la participation des populations locales, comme facteur de changement des mentalités traditionnelles, en vue d'une adhésion aux impératifs de la modernisation (Lebel, 1967) ${ }^{12}$. 
La signification que les aménagistes du BAEQ donnent à l'animation sociale est donc bien différente de celle que lui attribueront les organisateurs communautaires. Elle est pour les premiers un moyen non directif de faire passer leurs solutions, alors qu'elle représente pour les seconds une pédagogie de potentialisation des milieux populaires. Les techniques d'animation des groupes de travail que les organisateurs communautaires empruntent à l'éducation des adultes (Beaugrand-Champagne, 1968 ; Doray, 1967), qui se constitue alors à la marge de l'institution éducative, sont réinterprétées en fonction de cette visée (Blondin, 1970 : 303-307 ; Houle, 1972).

Malgré ces disparités de signification, I' « animation sociale » est bien le terme de ralliement dans lequel se reconnaissent les diverses expériences d'organisation communautaire qui s'enclenchent ou se poursuivent à Montréal ou en région, entre 1963 et 1970, sur des bases structurelles et culturelles comparables à celles évoquées ici. Les marges institutionnelles d'où partent les expériences se diversifient, cependant. À Saint-Jérôme, sur la marge où l'action catholique ouvrière croise l'institution syndicale, le chanoine et sociologue Jacques Grand'Maison anime, à compter de 1963, un « comité de travailleurs de différentes usines » (Grand'Maison, 1967 : 89). À Montréal, à compter de 1964, le Projet de réaménagement social et urbain (P.R.S.U.), financé par le University Settlement de l'Université McGill, se donne un volet d'animation sociale et développe des actions sur le logement, les loisirs, la santé et la sécurité du revenu (Boivin, $1988: 22-24)^{13}$. En 1965, naissent les Associations coopératives d'économie familiale (ACEF) qui sont le prolongement sur une base autonome des interventions de la Confédération des syndicats nationaux sur les problèmes d'endettement (René et Panet-Raymond, 1984 : 16 ; Fortin et Roland, 1980 : 5-6). À Québec, à compter de 1966, c'est un travalleur social de l'agence diocésaine de service social, le Service familial de Québec, qui intervient dans le quartier Saint-Roch, avec le curé de la principale paroisse du quartier. En 1967, un deuxième travailleur social se joint à lui. Il est engagé par la Coopérative d'habitation du Québec métropolitain pour travailler en priorité au développement d'un projet de logements coopératifs. Les deux travailleurs sociaux définissent leur action dans le courant de l'animation sociale (Doré, 1978 : 154-155, 325-326). En 1966, dans la foulée de la " guerre à la pauvreté » aux États-Unis, la Chambre des communes du Canada adopte la Loi sur la Compagnie des jeunes Canadiens dont les activités débutent à Montréal en 1968. Ses " volontaires » contribuent à de nombreuses initiatives, en vue de la mise sur pied de "comités de citoyens » et de « comités ouvriers » (McGraw, 1978 : 98-100). L'Union générale des étudiants du Québec (UGEQ) et le gouvernement du Québec collaborent pour mettre sur pied son équivalent québécois, les Travailleurs étudiants du Québec (TEQ) qui deviendront par la suite l'Action sociale jeunesse (Favreau, 1989 : 17). En 1968, à Hull, I'animation sociale débute à partir des préoccupations pastorales de l'Église diocésaine et du besoin ressenti par des 
travailleurs sociaux « de sortir de l'impasse du casework» (Poirier, 1986 : 20, 25-26).

Pour tous les intervenants et toutes les intervenantes issus de ces diverses marges institutionnelles et d'autres comparables, l'animation sociale est le mouvement culture ${ }^{14}$ qui fonde la tension de solidarité qui s'établit entre eux et elles, d'une part, en tant que fraction marginale de la petite bourgeoisie intellectuelle montante, et les classes populaires, d'autre part. Les groupes qui naissent de leur intervention, que ce soit en milieu rural ou en milieu urbain, ont en commun d'être véritablement des groupes de personnes de classe populaire : ouvriers et ouvrières, employées et employés exécutants, petits agriculteurs et agricultrices, exclus et exclues du marché du travail issus des précédentes catégories. En ce sens, le terme " groupes populaires » qui leur sera attribué dans les années 70 est juste et rigoureux d'un point de vue sociologique. Il s'agit d'une action collective dans laquelle les classes populaires sont vraiment actrices sociales et, de ce point de vue, d'un mouvement proche du mouvement ouvrier historique. Mais, en même temps, les liens qui se tissent entre les groupes qui composent ce mouvement populaire le différencient du mouvement ouvrier par la structure de réseau informel qu'ils adoptent et qui annonce celle des mouvements sociaux à venir (féminisme, écologisme, pacifisme).

L'expansion, la diversification et la politisation du mouvement populaire ont comme corollaire sa distanciation à l'égard du réseau institutionnel des services sociaux. La diversification des disciplines de formation des animateurs et animatrices accroît la distance déjà prise, dès le moment fondateur, entre I'organisation communautaire et la profession du service social (Doré, 1985 : 212-213). Des animateurs et animatrices formés en service social, et éventuellement rattachés à des organismes du réseau institutionnel des services sociaux, jouent un rôle important dans ce mouvement, mais la culture professionnelle qu'ils et elles se construisent dans l'action diffère profondément de celle véhiculée alors dans les sevices sociaux institutionnalisés.

Une des formes organisationnelles issues du mouvement populaire marquera pourtant profondément la transition vers la réforme des services de santé et des services sociaux de 1971. Cette forme organisationnelle est la clinique communautaire de quartier. Dans une monographie qui évoque avec beaucoup d'intensité et de vérité la tension éthico-culturelle et les rapports sociopolitiques qui ont présidé à la naissance de l'une d'entre elles, la Clinique des citoyens de Saint-Jacques, à Montréal, Robert Boivin (1988) nous fait suivre son parcours depuis 1968 jusqu'à son intégration dans le réseau public. Sans qu'on dispose d'une étude socio-historique bien documentée sur la dynamique politico-institutionnelle qui a conduit à cette option, il est généralement admis que les cliniques communautaires de quartier ont constitué un modèle de référence pour la conception de l'établissement public de 
base de la réforme de 1971, le Centre local de services communautaires (CLSC). Après s'être développé dans une explosion à l'extérieur du cadre institutionnel, le mouvement déclenché par l'organisation communautaire naissante se trouvera soumis à une implosion vers lui, dans le nouveau contexte structurel introduit par la réforme de 1971.

\section{L'INSTITUTIONNALISATION DANS LES SERVICES SOCIAUX PUBLICS ET LA RÉSISTANCE DE L'IDENTITÉ, DANS LE CONTEXTE DE LA PREMIÈRE RÉFORME (1971-1990)}

La Loi sur les services de santé et les services sociaux sanctionnée le 24 décembre 1971 institue le « centre local de services communautaires » et le définit comme " une installation autre qu'un cabinet privé de professionnel où on assure à la communauté des services de prévention et d'action sanitaires et sociales » (Québec, 1971 : art. 1,g). Un document interne du Ministère (alors ministère des Affaires sociales) prévoit que les territoires de CLSC devront répondre aux normes suivantes : desservir un bassin minimal de 10000 personnes en milieu rural et de 30000 en milieu urbain (MAS, 1971 : 9). Le concept de territoire de CLSC serre donc de très près la définition de la communauté locale qui a servi de cadre d'action aux expériences d'animation sociale de la décennie précédente. La participation active des personnes concernées, qui est un des principes clés de spécification de l'organisation communautaire comme méthode de travail social, trouve une forme d'institutionnalisation dans l'article de la loi qui prévoit que cinq des onze membres votants du conseil d'administration du CLSC seront des personnes usagères de ses services (Québec, 1971 : art. 50) ${ }^{15}$.

Au-delà des dispositions explicites de la loi, la mise en œuvre de la réforme instaure une nouvelle organisation du travail qui produit une conséquence majeure pour l'organisation communautaire. Dans le processus de syndicalisation et de négociation collective qui suit l'implantation des premiers CLSC, la fonction d'organisateur ou organisatrice communautaire est instituée dans un titre d'emploi qui est un attribut spécifique ${ }^{16}$. Contrairement à ce qui se passait dans les services sociaux privés du début des années 60, ce titre d'emploi est bien démarqué de l'administration des services sociaux, dont les agents et agentes sont clairement définis comme des cadres et, de ce fait, ne font pas partie de l'unité d'accréditation syndicale. L'organisateur ou I'organisatrice communautaire dans un CLSC est une " personne qui fait I'identification et l'analyse des besoins de la population avec des groupes concernés. Conçoit, coordonne et actualise des programmes d'organisation communautaire afin de répondre aux besoins du milieu et de promouvoir son développement. Agit comme personne-ressource auprès des groupes » 
(FAS, 1990-1991 : 220). Le titre d'emploi de « travailleur ou travailleuse communautaire » est aussi institué pour les personnes ayant une formation de niveau collégial ou l'équivalent, avec une définition de fonction axée sur l'exécution des programmes d'organisation communautaire, plutôt que sur leur conception et leur coordination.

Malgré leurs limites, et notamment l'imprécision de l'expression " programmes d'organisation communautaire », ces définitions comportent l'avantage de confirmer l'organisation communautaire en tant que pratique orientée vers les groupes des milieux où sont implantés les CLSC, et fournissent une base d'argumentation pour la défense de la spécificité de la pratique. En même temps, par ailleurs, l'institutionnalisation de l'organisation communautaire dans une fonction professionnelle du réseau public introduit une dualité dans les conditions de mise en œuvre de la pratique, entre le réseau public lui-même et le mouvement qui continue à exister en dehors de lui. Au cours de la période 1971-1980, le développement de l'identité passera pour l'organisation communautaire par le dépassement de cette dualité.

\section{Le dépassement de la dualité réseau / hors réseau (1971-1980)}

Au cours de cette période, le mouvement populaire que l'organisation communautaire naissante a contribué à déclencher poursuit son élaboration grâce à l'action combinée de militantes et militants accrochés à diverses marges institutionnelles et d'organisateurs et organisatrices communautaires du réseau public. Pour peu que ces derniers aient un principe d'identité bien articulé et une bonne dose d'autonomie professionnelle, ils et elles réussissent habituellement à se donner la marge de manœuvre qui leur permet d'insérer leur pratique dans la dynamique des groupes du milieu en mouvement. Le terme d' ' animation sociale » tombe en désuétude, dans des circonstances qu'il resterait à élucider, mais le mouvement qui s'en est inspiré poursuit son expansion. On parle moins de " comités de citoyens » et davantage de " groupes populaires ». L'expansion est géographique et touche pratiquement toutes les régions du Québec. Dans le Bas- Saint-Laurent et la Gaspésie, par exemple, au cœur de l'impasse à laquelle a conduit l'expérience avortée de planification régionale du BAEQ, les Opérations-Dignité (Dionne, 1979 : 51 ; JAL, 1979 ; 64-65) sont la première manifestation d'un mouvement qui connaîtra jusqu'à aujourd'hui de multiples expressions et résurgences. À Sherbrooke, une dizaine de comités naissent de l'action de quelques vicaires ouverts aux questions sociales, de religieuses appuyées par des étudiants et étudiantes en théologie, et d'étudiants et étudiantes en service social (Deslauriers et Pouliot, 1982 : 13). Aux marges institutionnelles qui continuent à accueillir les organisateurs et organisatrices communautaires 
s'ajoutent, en 1973, les Services éducatifs d'aide personnelle et d'action communautaire (SEAPAC), dans les services d'éducation aux adultes des commissions scolaires régionales (Pelletier, 1973). À la même époque, des groupes populaires commencent à se regrouper, au niveau régional puis national, selon leurs rapports avec le ministère de l'Éducation en tant que bailleur de fonds de l'éducation populaire autonome. Ces regroupements déboucheront sur le Mouvement d'éducation populaire et d'action communautaire du Québec (MEPACQ, 1984). L'expansion se traduit aussi par une diversification des champs de pratique : sécurité du revenu avec les groupes de défense des droits des personnes assistées sociales (René et Panet-Raymond, 1984 : 58 ; Ventelou, 1983 ; Gaudreau, 1983) et les mouvements Action-Chômage (Girard, 1989 ; Auclair et Béland, 1983), développement local et régional avec les entreprises communautaires et les coopératives dans l'est du Québec (Lévesque, 1979), petite enfance avec les garderies sans but lucratif contrôlées par les parents usagers (Labrie, 1985 ; Lalonde-Graton, 1985), emploi avec les coopératives de travail et les expériences d'autogestion (Centre, 1975 ; Boucher, 1982 ; AA.VV., 1985), culture avec les médias communautaires (Larose, 1983 ; Vallières, 1985) et le théâtre d'intervention autogéré (Théâtre, 1982, 1987), gérontologie avec l'Association québécoise pour la défense des droits des retraités et préretraités (AQDR) (Laforest, 1983), etc.

$C^{\prime}$ est au point de jonction entre ce mouvement et leurs marges institutionnelles respectives que les organisateurs et organisatrices communautaires poursuivent alors leur réflexion sur leur pratique. En 1977, à la suite d'une série de rencontres à l'initiative d'un noyau de professeurs de l'École de service social de l'Université Laval, est fondé le Regroupement des organisateurs communautaires du Québec qui deviendra, en 1983, le Collectif québécois de conscientisation. Le ROCQ est ouvert à tous ceux et celles qui ont une pratique d'animation des collectivités et se reconnaissent dans son manifeste. Le manifeste de 1977 analyse la situation de classe et la situation professionnelle des organisateurs et organisatrices communautaires et avance une stratégie consistant à lier les initiatives coopératives et les luttes revendicatives au développement de la conscience et de la lutte politiques (ROCQ, 1979 [1977]). Le ROCQ est avant tout un collectif autogéré de formation continue. Ses thèmes de formation des années 70 sont l'évaluation des luttes sociales, les rapports entre luttes populaires et groupes politiques, la conscientisation, I'organisation communautaire en milieu rural et la question nationale (ROCQ-CQC, 1987 : 41).

En 1978, le ROCQ mène une enquête sur la situation d'ensemble de l'organisation communautaire au Québec, avec la collaboration de ses coordonnateurs régionaux. II ressort de cette enquête que le Québec compte alors 492 organisateurs et organisatrices communautaires ou personnes salariées 
accomplissant des tâches de même nature. Les groupes ou associations avec lesquels ils travaillent sont, par ordre d'importance, les comités de citoyens, les garderies populaires, les journaux populaires, les groupes de pression de personnes âgées, les associations de loisirs, les clubs de l'Âge d'or, les associations de locataires, les comptoirs alimentaires, les groupes de bénévoles, les maisons ou centres communautaires, les comités de liaison entre mouvements, les syndicats ouvriers, les comités d'école ou de parents, les comités de paroisse, les groupes d'action de personnes handicapées, les coopératives $d^{\prime}$ habitation, les maisons ou centres de femmes, les projets culturels populaires, les associations de locataires de logements municipaux, les cafés-rencontres, les conseils régionaux de développement et, enfin, les partis politiques municipaux (Doré et Larose, 1979).

Il est très important de noter aussi que, déjà à cette époque, 13,6 \% des répondantes et répondants qui sont identifiés comme organisateurs et organisatrices communautaires ont en fait une pratique de « marketing et développement de services » ou encore de " coordination de groupes de bénévoles » (Doré et Larose, 1989 : 92) plutôt qu'une pratique correspondant à la différence spécifique de l'organisation communautaire : intervention collective basée sur la participation active des personnes qui vivent les situations en cause. Sur cette base, le nombre d'organisateurs et d'organisatrices communautaires devrait être ramené à environ 425. De plus, quand on isole les répondants et répondantes des services sociaux publics des autres répondants et répondantes, la proportion de gestionnaires monte à 17,7\%. Ces statistiques nous laissent entrevoir qu'une tendance est alors à l'œuvre dans les CLSC et qu'elle ne va pas dans le sens d'une confirmation de la différence spécifique de l'organisation communautaire. Dans la période qui suivra, cette tendance prendra du relief et du contenu avec la montée du discours sur « I'approche communautaire ». Plus que jamais auparavant, depuis l'expérience fondatrice qui lui a conféré son identité, I'organisation communautaire verra celle-ci mise en question par l'hégémonie montante d'un discours institutionnel équivoque qui emploie un terme commun pour nommer une pratique différente.

\section{La persévérance de l'identité face à l'" approche communautaire » et au « néo-bénévolat » (1981-1990)}

En 1981, est publié I'essai du sociologue français Pierre Rosanvallon sur la crise de l'État-providence (Rosanvallon, 1981) et, en 1982, le rapport du groupe de travail présidé par le travailleur social anglais Peter M. Barclay, sur le rôle et les tâches des travailleurs sociaux (Barclay, 1982). Les deux auteurs préconisent une voie de sortie de l'État-providence, sous le titre de « post-social-démocratie » dans un cas (Rosanvallon, 1981 : 136-138) et 
$d^{\prime}$ « approche communautaire » dans I'autre (Barclay, $\left.1982: 104\right)$. Tous deux préconisent la stabilisation de l'État-providence à son niveau du début des années 80 (Rosanvallon, 1981 : 137 ; Barclay, 1982 : 83-84), en comptant sur les capacités autonomes des personnes, des familles, des groupes de voisinage et des réseaux d'entraide locaux pour résorber la nouvelle demande sociale (Rosanvallon, 1981 ; 112, 117-118 ; Barclay, 1982 : 198-217). Les deux ouvrages vont directement influencer l'idéologie et la stratégie de la Fédération des centres locaux de services communautaires, en relation avec celles du ministère de la Santé et des Services sociaux, pour le reste de la décennie (Gingras, 1988 : 9-11, 24-26 ; Bourque, 1989 :13-21 ; Lachapelle, 1990 : 6-18). Après la publication du rapport Barclay, le terme $d^{\prime}$ " approche communautaire ", déjà en usage dans le vocabulaire des CLSC, change de définition, pour adopter celle qui lui est attribuée en Angleterre. II ne renvoie plus à la promotion de l'action communautaire autonome contre les causes des problèmes sociaux et de santé, mais plutôt au recours aux réseaux d'aidantes et aidants "naturels », c'est-à-dire la famille, les amis et amies et puis le bénévolat, dans une perspective de contrôle des dépenses publiques. La capacité présumée des CLSC de mobiliser les ressources communautaires dans le sens indiqué est l'argument de poids que la Fédération met dans la balance du Ministère, pour la faire pencher en faveur de la survie et du développement de son réseau.

Dans cette stratégie institutionnelle, une équivoque que l'on croyait dissipée depuis l'époque de l'animation sociale refait surface. Un mandat de gestion des services sociaux est dévolu à l'organisation communautaire ; résurgence d'autant plus inattendue que contrairement à autrefois, l'administration est maintenant une spécialisation clairement définie et bien différenciée de l'organisation communautaire. En réalité, la résurgence est rendue possible parce qu'elle apparaît sous des traits nouveaux. Les organisateurs et organisatrices communautaires sont conviés à agir comme gestionnaires de second niveau, coordonnateurs et coordonnatrices des contributions des ressources bénévoles, sous la gouverne des administrateurs et administratrices en titre, responsables des ressources humaines professionnelles. À cet égard, la formalisation idéologique ne vient qu'accréditer et renforcer la tendance décelée dans la période précédente. En 1988, selon les données d'une enquête effectuée dans le réseau des CLSC (AA.VV., 1989), 33,1 \% des 357 personnes «qui occupent un poste d'organisateur ou de travailleur communautaire en CLSC ou qui sont reconnues dans leur milieu pour faire un travail assimilable sans avoir le titre » seront en réalité affectées à l' « organisation de services ", c'est-à-dire qu'elles " appuient ou initient des services qui visent l'amélioration des conditions de vie d'une population ; travaillant parfois en collaboration avec d'autres institutions ou des groupes bénévoles ; si nécessaire, contribuent à la mise sur pied du groupe bénévole » (AA.VV., $1989: 13$ et 83$)$. 
La pression à la complémentarité de l'action communautaire autonome à l'égard du secteur public est en même temps beaucoup plus marquée qu'auparavant. Plusieurs des marges institutionnelles sur lesquelles s'était greffée l'organisation communautaire ont dépéri et sont disparues dès les années 70. La Compagnie des jeunes Canadiens et l'Action sociale jeunesse ont été abolies dans ces années. L'Action catholique ouvrière a décliné dans un contexte de sécularisation. Dans les années 80, les centres de services sociaux qui, dans la réforme de 1971, avaient remplacé les services sociaux diocésains ont éliminé leurs derniers postes d'organisation communautaire. Les syndicats se sont repliés sur leur champ prioritaire, celui du travail. Les Services éducatifs $d^{\prime}$ 'aide personnelle et d'action communautaire (SEAPAC) sont disparus dans les commissions scolaires régionales. À la suite de ces changements, le rapport des groupes communautaires aux institutions a tendu à se polariser sur le ministère de l'Éducation pour le volet « éducation populaire » et sur le ministère de la Santé et des Services sociaux et les CLSC pour le reste.

Beaucoup de pratiques antérieurement axées sur la participation des collectivités locales à la défense de leurs droits et à la gestion de leurs services ont évolué vers la seule prestation de services par des bénévoles ou des permanents et permanentes en emploi précaire, dans un régime de soustraitance avec l'État (Panet-Raymond, 1985). Plusieurs des organismes communautaires issus des mouvements de la précédente décennie et aujourd'hui financés par le ministère de la Santé et des Services sociaux sont en réalité des services sociaux privés sans but lucratif d'un type nouveau, mais qui n'actualisent plus la différence spécifique de l'organisation communautaire en ce qui a trait à la participation active des personnes aux prises avec les problèmes, en vue d'un changement social et non seulement personnel. Cette situation coïncide avec un renouveau du bénévolat, à partir de facteurs endogènes insuffisament expliqués jusqu'à aujourd'hui, que l'État a tout de même bien saisi et qu'il encourage par des incitations morales et financières (Laforest et Redjeb, $1989: 17-42)^{17}$. Une confusion est d'ailleurs entretenue entre bénévolat et action communautaire, alors que les deux n'ont en commun que la gratuité et l'utilité du geste, et que l'action communautaire se distingue clairement du bénévolat par l'engagement prioritaire des personnes concernées, et non de celles qui veulent aider, pour un changement prioritairement social et non personnel; ce dernier point marque la différence d'avec le groupe d'entraide que Laforest et Redjeb incluent comme une des catégories du « néo-bénévolat» (1989; 39-40).

Au paroxysme de cette équivoque, des groupes se réorientent ou se fondent dans un but qui est un déni du sens de l'organisation communautaire, aussi bien que du travail social et du bénévolat. Ce but consiste à définir son existence, en fonction des créneaux de complémentarité définis par l'État, en comptant sur la contribution du " bénévolat forcé » des assistées sociales et 
assistés sociaux, à travers les programmes d'employabilité, en particulier le programme EXTRA, et éventuellement avec la collaboration d'un CLSC qui donnera un mandat à cet effet à un organisateur ou à une organisatrice communautaire (Gmati et Robitaille, 1991 ; Soulié, 1991 ; Le Soleil, 1991) ${ }^{18}$.

En lieu et place des termes " groupes populaires » et " mouvement populaire » qui tendent à tomber en désuétude à leur tour, dans une dynamique socio-sémantique qu'il resterait à expliquer, les termes « groupes communautaires » et "mouvements communautaires», puis " organismes communautaires »sont utilisés pour renvoyer aux composantes pourtant très diverses de cet amalgame. Est-il possible de dépasser la confusion qui en ressort, pour situer la place de l'organisation communautaire au cours de cette période, aussi bien dans le réseau public que dans celui dit communautaire?

En se livrant à un exercice d'extrapolation, on peut arriver à des chiffres plausibles, bien que discutables, qui nous permettent de situer la présence quantative de l'organisation communautaire, en incluant sous ce terme toutes les personnes exerçant, à temps plein ou à temps partiel, une pratique salariée d'animation des collectivités. J'arrive pour ma part à une estimation qui se situe autour de $650^{19}$ à 23 . Si l'on compare ce résultat aux 425 salariées et salariés recensés en 1978, l'augmentation de 225 en chiffres absolus suppose une hausse de 53,7\% sur dix ans. Si cette extrapolation est juste, on assisterait donc à une lente progression sur le plan quantitatif, mais à laquelle ne semble pas correspondre une polarisation identitaire d'amplitude comparable, sur le plan qualitatif.

Entre 1964 et 1970, alors que le Québec ne comptait que quelques dizaines de praticiens et praticiennes de l'organisation communautaire, $\mathrm{I}^{\prime}$ « animation sociale » a investi la pratique d'une très forte polarité identitaire. Entre 1971 et 1980, alors qu'ils étaient quelques centaines, la référence aux " groupes populaires » a pris la relève avec une égale intensité. Après 1981, alors que la progression numérique continue, force est de constater l'absence d'une polarité identitaire comparable. Les termes de " groupes communautaires ", " mouvement communautaire » et " organismes communautaires » ont autant servi jusqu'à maintenant à entretenir une équivoque qu'à construire une référence identitaire catalysatrice. En réalité, pourtant, des pôles d'identité se sont constitués ou sont entrés en gestation au cours de cette période, à partir des marges diverses où évoluent toutes les catégories de personnes exerçant une pratique d'animation des collectivités.

En 1983, le Regroupement des organisateurs communautaires du Québec est devenu le Collectif québécois de conscientisation (ROCQ-CQC, 1987 : 21-22 ; Comeau, 1989). Même si la très forte charge identitaire de cette association entre organisation communautaire et conscientisation fait tiquer quelques auteurs (Alary, 1988 : 78-79 ; Hurtubise, 1991 : 155-159), elle n'en présente pas moins les traits d'une réaffirmation de l'intention 
originelle, d'une réactivation du moment fondateur, ce que le sociologue italien Francesco Alberoni appellerait une "élaboration éthique » de ce moment (Alberoni, 1989 : 318-328). On retrouve une analogie frappante entre la conscientisation définie comme " la réalisation d'une option politique dans une stratégie culturelle d'alliance avec la classe populaire » (CQC, 1983) et certains textes fondateurs de Blondin, notamment celui où il met en évidence la coupure culturelle entre les travailleurs sociaux et les milieux de pauvreté où ils interviennent (Blondin, 1989 [1966]). Le Collectif compte une centaine de membres pour qui il constitue un lieu de ressourcement et d'autoformation. Outre la connivence avec l'approche de conscientisation, la base d'adhésion est l'engagement dans une action collective, quels qu'en soient le champ de pratique et la forme organisationnelle. Environ dix pour cent de ses membres sont rattachés au réseau public, les autres appartenant à des groupes communautaires autonomes. Vingt pour cent d'entre eux sont des militants et des militantes de classe populaire formés dans I'action ${ }^{24}$. Entre 1977 et 1987, trois cents praticiens et praticiennes de l'organisation communautaire sont passés par les activités de formation du ROCQ-CQC (ROCQCQC, 1987 : 26-27), sans compter la contribution de membres du CQC à des activités de formation organisées en dehors du CQC, telles que la formation du personnel du réseau public (Ampleman, 1987) ainsi que des étudiants et étudiantes en milieu universitaire (Université Laval, 1991-1992), ou encore la participation comme formateurs ou formatrices à des colloques ou séminaires de formation, comme le Forum sur les inégalités socio-économiques et la santé tenu en 1989 (Martin, 1990 : 37, 39-40).

Toute la décennie 1981-1990 a aussi été marquée par la stratégie menée par des groupes communautaires pour affirmer leur différence face à l'État, dans le contexte équivoque $d^{\prime}$ " approche communautaire » précédemment décrit. Dans un article récent, Larochelle et Robichaud (1991 : 659-671) rendent bien compte de la dynamique de ce rapport. Ce qui nous intéresse dans l'analyse de celui-ci, c'est la place faite par les groupes communautaires, dans la définition qu'ils donnent d'eux-mêmes, à la participation des personnes aux prises avec les problèmes sociaux, dans un but de changement social. Cette spécificité de l'organisation communautaire est présente dans le discours des groupes, bien qu'elle n'y occupe pas une place prépondérante. Dans le cadre des travaux qu'elle a réalisés pour le compte de la Commission d'enquête sur les services de santé et les services sociaux (Rochon, 1988), et qui I'ont mise en contact avec « des intervenantes et intervenants œuvrant dans 17 milieux communautaires de la région métropolitaine », Lamoureux a constaté que : « Rendre socialement utiles et politiquement actives des collectivités entières de marginalisés/es, d'exclus/es, de laissés/es pour compte est la mission essentielle $d^{\prime}$ 'une grande partie de la mouvance communautaire » (Lamoureux, $1988: 161$ et 170 ; je souligne). Dans le mémoire qu'il a présenté au nom du Regroupement des organismes communautaires 
jeunesse du Montréal métropolitain (ROCJMM) à la Commission parlementaire qui étudiait l'avant-projet de loi sur les services de santé et les services sociaux, Parazelli avance que : "Au-delà des diversités de moyens d'intervention et d'approche, les organismes communautaires ont, de façon générale, les mêmes objectifs de fond : la prise en charge des citoyennes et citoyens par eux-mêmes, la socialisation (volontaire) des rapports humains entre les citoyens et leur communauté, l'action sur les causes des problèmes sociaux et de santé »(Parazelli, $1990: 180$; je souligne).

Au cours des années 80, la polarisation identitaire des groupes communautaires par champ de pratique s'est accentuée. Aux regroupements qui marquent la continuité avec la décennie précédente s'en sont ajoutés de nouveaux dans une grande diversité de références identitaires : Regroupement des centres de femmes du Québec, Regroupement provincial des maisons d'hébergement et de transition pour femmes violentées, Regroupement des ressources alternatives en santé mentale du Québec, Regroupement des organismes provinciaux de personnes handicapées, Regroupement des maisons de jeunes du Québec (RMJQ), etc.

En 1986, un colloque organisé à l'initiative de la Corporation de développement communautaire des Bois-Francs et ouvert à une grande diversité de groupes de toutes les régions du Québec (AA. VV., 1987 : 233-238) cherchera à transcender ces références sectorielles pour engager ses participantes et participants dans une réflexion sur l'identité commune. L'animatrice du comité de synthèse résume le résultat de cette réflexion dans les termes suivants : «Ce qui nous rassemble et nous distingue par rapport à l'approche dite " communautaire » du gouvernement... : le souci d'un fonctionnement démocratique... le communautaire est porteur de valeurs alternatives dans lesquelles la prise en charge et l'autonomie, individuelle et collective, prennent une grande importance... l'importance de se donner une formation... rôle d'éclaireur au niveau de l'identification des besoins... la volonté d'un changement social et la recherche d'un projet de société différente » (Daniel, 1987). Aucune structure permanente ne naîtra de ce colloque. Par ailleurs, une Coalition des organismes communautaires du Québec avait déjà vu le jour en 1985 (Parazelli, 1988 ; COCQ, 1988) et, en 1990, une nouvelle coalition de regroupements d'organismes, dite Table MSSS, sera fondée dans le cadre des débats sur le projet de loi sur la santé et les services sociaux (Fournier, $1991: 11$ ).

Pour sa part, le colloque de 1986 aura été une occasion de rencontre pour des intervenantes et intervenants en organisation communautaire de plusieurs CLSC. Un comité en sortira qui lancera un bulletin de liaison ${ }^{25}$ et organisera un colloque, en 1988, à l'intention des organisateurs, organisatrices, travailleurs et travailleuses communautaires de CLSC (Hurtubise et Paré, 1989). Le Regroupement québécois des intervenants et intervenantes en 
action communautaire en CLSC (RQIIAC) y sera fondé. En 1990, au moment du deuxième colloque, il comptera 100 membres en règle sur un total possible de $350^{26}$. Aux références identitaires fondées sur le modèle de pratique (CQC), le bailleur de fonds institutionnel (MEPACQ) ou le champ de pratique (les divers regroupements sectoriels), le RQIIAC vient ajouter la référence identitaire fondée sur l'employeur institutionnel. Comme dans le cas de l'identité fondée sur le champ de pratique ou le bailleur de fonds, la recherche de cohésion doit y composer avec la diversité de pratiques que recouvre l'unité de terminologie, notamment celles qui s'écartent de la différence spécifique de l'organisation communautaire en tant que pratique axée sur la participation et le changement social. Cette préoccupation se reflète dans le programme provisoire du troisième colloque. En introduction à la thématique, les membres du comité organisateur y affirment que «I'action communautaire en CLSC doit être abordée de façon à rallier le plus d'intervenantes et d'intervenants possible plutôt que de provoquer des débats idéologiques. Nous ne pensons pas, à titre d'illustration, qu'il soit souhaitable de travailler sur les différents modèles d'action communautaire auxquels nous nous référons : ce serait mettre l'emphase sur ce qui nous distingue les uns des autres » (RQIIAC, 1991 : 2). L'institutionnalisation de la pratique se traduit ici par un déplacement de la radicalité de l'identité existentielle, c'est-à-dire celle reliée à l'expérience fondatrice et à son élaboration en mouvement, vers les compromis de l'identité institutionnalisée ; ce qui reste cependant compatible avec une forte adhésion à l'identité existentielle chez beaucoup de membres du RQIIAC, comme en témoignent plusieurs articles publiés dans son bulletin de liaison.

En 1990, deux membres du RQIIAC ont participé à un groupe de travail de la Fédération des CLSC sur l'« action communautaire » (Vallée, 1991). Le document qui est sorti des travaux de ce comité est avant tout un texte de légitimation institutionnelle de I' " action communautaire ", après une période, celle de l'« approche communautaire » des années 80 , où elle avait été mise sous le boisseau (FCLSC, 1991 : 2-3). Le document donne aux organisateurs, organisatrices, travailleurs et travailleuses communautaires de CLSC des références institutionnelles auxquelles s'arrimer, pour justifier leur pratique, tout en perpétuant les ambiguïtés et les flous qui traversent la courte histoire des idées dans les CLSC.

Comme I' « approche communautaire ", I'« action communautaire » est I'affaire de tout le monde dans le CLSC, et non la responsabilité principale de ceux et celles qui sont censés avoir été engagés avec la formation pour la pratiquer (FCLSC, $1991: 4,15,29,31,38$ ), tout en convenant que « les CLSC doivent pouvoir faire appel à une équipe minimale $d^{\prime}$ intervenants en action communautaire » (30). Le choix du terme " action communautaire » au lieu du terme «organisation communautaire » découle sans doute de cette 
préoccupation, alors qu'en toute rigueur sémantique on aurait dû le réserver à l'action de la communauté et non à l'intervention professionnelle avec la communauté. La définition donnée à l'action communautaire, en tant que " mode d'intervention qui vise ultimement à créer les conditions favorables à la santé » (9), n'est pas une définition comportant un niveau d'universalité qui la rende partageable avec d'autres organisations que celle du réseau sociosanitaire. La santé y est par ailleurs définie en termes tellement larges qu'elle peut inclure les problèmes liés aux structures socio-économiques, politiques et socioculturelles qu'on se refuse à nommer par leur nom (10-11). L'ambivalence entre " action communautaire », au sens d'organisation communautaire comme pratique participative, et " action communautaire", au sens $\mathrm{d}^{\prime}$ " approche communautaire » ou recours aux personnes et groupes de milieu pour l'aide individualisée, est entretenue $(14)^{27}$. La place prépondérante accordée au "support aux ressources », de même qu'à leur création et concertation, dans l'identification des « types d'action en action communautaire » $(15-17,21)$, est d'ailleurs beaucoup plus en accord avec le deuxième pôle de l'ambivalence qu'avec le premier. Un accent marqué est mis sur le contrôle politique de l'action communautaire par l'institution (18-19).

En somme, dans les CLSC, à la veille de la deuxième réforme des services de santé et des services sociaux, un nouveau discours équivoque prend le relais du discours usé de l'approche communautaire des années 80 . On n'y trouve rien de bien substantiel pour nourrir la charge éthique qui porte les organisateurs et organisatrices communautaires à la rencontre des milieux populaires et des groupes marginalisés : tout au plus quelques nouveaux repères institutionnels pour défendre la pratique.

Si l'on résume les traits saillants de la période 1981-1990, on peut constater que malgré l'équivoque créée par la conception étatique de l'« approche communautaire » et l'émergence d'un " néo-bénévolat », un progrès quantitatif et une certaine persévérance de l'identité sont observables en organisation communautaire. II s'agit toutefois d'une identité multipolaire dont on peut appréhender qu'aucun des pôles ne soit en mesure de s'imposer comme catalyseur de l'ensemble, face aux défis introduits par la nouvelle réforme des services sociaux.

\section{L'INSTITUTIONNALISATION DANS LES « ORGANISMES COMMUNAUTAIRES » ET LE DÉFI DE LA SURVIE DE L'IDENTITÉ, DANS LE CONTEXTE DE LA DEUXIÈME RÉFORME (1991- ）}

À l'encontre de l'étatisation presque intégrale de 1971, la réforme de 1991 marque le retour des services sociaux privés sans but lucratif dans la stratégie de l'État à l'égard des problèmes sociaux. La Loi sur les services de santé 
et les services sociaux et modifiant diverses dispositions législatives, sanctionnée le 4 septembre 1991, leur confère une reconnaissance et une légitimité qu'ils avaient perdues avec la première réforme. Le langage que les plus dynamiques d'entre eux ont su imposer, au cours des dix dernières années, dans la foulée des mouvements sociaux d'où ils sont sortis, celui des « organismes communautaires », devient le code culturel dans lequel ils sont tous nommés. L'implosion institutionnalisante est nette et bien affirmée.

Dans la présente loi, on entend par " organisme communautaire » une personne morale constituée en vertu d'une loi du Québec à des fins non lucratives dont les affaires sont administrées par un conseil d'administration composé majoritairement d'utilisateurs des services de l'organisme ou de membres de la communauté qu'il dessert et dont les activités sont reliées au domaine de la santé et des services sociaux.

Un organisme communautaire qui reçoit une subvention en vertu du présent titre définit librement ses orientations, ses politiques et ses approches.

Une régie régionale peut... subventionner un organisme communautaire dans l'un ou l'autre des cas suivants :

1) s'il offre des services de prévention, d'aide et de soutien aux personnes de la région...

2) s'il exerce, au niveau de la région, des activités de promotion, de sensibilisation et de défense des droits et des intérêts des utilisateurs de ses services ou des usagers de services de santé ou de services sociaux de la région.

Une régie régionale peut également subventionner un organisme communautaire qui s'occupe, au niveau de la région, de la promotion de la santé et du développement social lorsque les plans régionaux d'organisation de services qu'elle a élaborés le prévoient (Québec, 1991 : 97-98).

La loi prévoit aussi que le Ministère exerce les mêmes fonctions à l'égard d'organismes s'occupant des mêmes questions pour l'ensemble du Québec, s'y ajoutant " des organismes communautaires qui exercent des activités répondant à des besoins nouveaux » et « des regroupements provinciaux d'organismes communautaires» (Québec, 1991 : 98).

Sur le plan formel, la loi fournit un fondement institutionnel aux demandes de financement des groupes communautaires, y compris à des fins de défense des droits ; un terme que le document de la Fédération des CLSC sur I'action communautaire n'ose même pas énoncer. Elle impose une réflexion stratégique autant aux groupes eux-mêmes qu'aux organisateurs et organisatrices communautaires qui les accompagnent dans leur cheminement, 
y compris ceux et celles des CLSC qui y font face à un défi de réalignement plus clair et mieux articulé que celui que leur propose la Fédération des CLSC.

Dès la Commission d'enquête sur les services de santé et les services sociaux, puis dans la période de l'avant-projet de loi, des groupes communautaires ont pressenti et affirmé les risques d'une intégration sous la tutelle régulatrice de l'État et cherché à définir les conditions d'un « partenariat véritable », à l'encontre d'une " complémentarité obligée » (Lamoureux, 1988 ; Guay, 1991a [1989] ; Parazelli, 1990 ; David, 1991). Au-delà des conjectures, une évaluation d'un projet-pilote réalisé dans les paramètres de la loi, avant que celle-ci ne soit adoptée, peut nous aider à en anticiper certains effets. Il s'agit du « bilan de l'expérience de participation de services en santé mentale »(Guay, 1991b). Parmi les éléments de ce bilan, il s'en trouve un qui interpelle particulièrement la définition de l'organisation communautaire, telle que nous I'avons exposée ici, dans son rapport existentiel à la continuité du moment fondateur, à travers la dynamique du mouvement qui en est issu.

Le manque total de pédagogie participative (...) a produit cette immense contradiction, écrit Guay : I'absence des usagers-ères du processus de planification des services en santé mentale alors que cette planification prétend se faire pour eux, alors que la politique de santé mentale les plaçait au centre. Cette situation amène les ressources alternatives à être en constante contradiction avec leurs valeurs, leurs approches, leur philosophie de base dont un des éléments principaux consiste à promouvoir la participation des usagers-ères à toute la vie associative et à toutes les instances décisionnelles de leur groupe (Guay, 1991b : 8-9).

Avec le retour, sous de nouvelles modalités culturelles et juridicopolitiques, des services sociaux privés sans lut lucratif sous la légitimité statutaire de l'État, l'organisation communautaire est-elle vouée à être réassimilée dans un mode de gestion, à l'instar de ce qu'elle était avant l'expérience fondatrice de I' « animation sociale » ? Le discours institutionnel des Morin (1962) et Laplante (1962) va-t-il réabsorber celui des Prud'homme (1961) et Blondin (1989 [1966], 1987, 1970, 1965) et de tous les autres qui leur ont succédé sur la voie de l'action collective avec des personnes marginalisées, pour le changement social?

Malgré des pôles identitaires où se manifestent avec des intensités variables une continuité et un renouvellement de l'expérience fondatrice en organisation communautaire, le lieu de l'action tend à se déplacer des bases sociales où se vivent les problèmes vers ces micro-institutions que sont devenus les « organismes communautaires », avec leur propre logique d'autoreproduction, en symbiose obligée avec l'État. Dans la nouvelle conjoncture créée par la réforme, les « organismes communautaires »sont exposés plus que jamais à devenir un système d'action pour lui-même, et non plus une médiation pour l'émergence de la solidarité des collectivités inorganisées, 
face aux enjeux économiques, politiques et culturels auxquels ils font face. Par la conjonction d'éléments structurels et culturels qui ont créé les conditions d'une expérience fondatrice, la marge des services sociaux a croisé pendant trente ans le champ social où surgissent de nouveaux sujets collectifs, parmi les populations réputées inaptes à le devenir. La pérennité de ce croisement n'a jamais été assurée. Une alliance s'est développée sur la ligne du risque, sur la frontière des classes et des cultures, en contact continu avec des situations-limites dans lesquelles elle pouvait se perdre. La conscience de ce risque d'évanescence est plus que jamais présente chez ceux et celles qui vivent l'organisation communautaire comme une médiation de la solidarité pour le changement.

La voie de la ré-appropriation et de la ré-affirmation de l'identité est connue, pour peu qu'on y porte attention. Elle est inscrite dans la trame de l'expérience acquise. Elle passe par un déplacement focal du centre de l'institution vers sa marge, de sa rationalité vers la réalité existentielle des collectivités aux prises avec des problèmes sociaux, expliqués non comme manifestations épidémiologiques, mais comme expression des rapports socio-économiques, sociopolitiques et socioculturels que nous entretenons entre nous, êtres humains de cette société. Ce retour aux bases sociales peut être lieu de ressourcement de la tension éthique qui nourrit l'action, dans le renouvellement cathartique ${ }^{28}$, c'est-à-dire ce double mouvement d'identification socioaffective (la " passion de la solidarité »: Doré, 1991a : 131-132) et de distanciation critique, un peu à l'image de cette animation sociale des débuts, à la fois engagement dans les milieux défavorisés et rationalisation de l'action collective. L'expérience fondatrice n'est pas que forme phénoménale disparue, et donc source de nostalgie. Elle est aussi, à un autre niveau, en tant $q^{\prime} u^{\prime}$ expérience fondamentale, structure métahistorique ${ }^{29}$ susceptible de se réactualiser dans des formes adaptées au temps présent. Et les institutions? et leur prégnance incontournable ? Elles restent, c'est vrai, le contexte avec lequel il faut inévitablement composer, la zone de résistance par rapport à laquelle il faut nécessairement se définir dans un rapport tactique. Mais celui ou celle qui se place délibérément, mais habilement, à leur marge et non en marge d'elles, ne doit pas désespérer de trouver en leur sein un écho à son engagement. Puisse cette parole d'Alberoni être vraie : « Les idéaux profonds, l'espérance enfouie au cœur de l'institution sont les mêmes que celles du moment fondateur » (Alberoni, $1989: 202$; je souligne). II me semble que cela pourrait se vérifier pour beaucoup $\mathrm{d}^{\prime}$ " organismes communautaires » et un certain nombre de CLSC. C'est dans l'action qu'on pourra leur permettre d'actualiser leur potentialités! 


\section{Notes}

1. Pour une définition plus complète et plus précise de l'organisation communautaire, voir : Doré, 1985 ; 1991 : 125, 132-136. Sur la définition des problèmes sociaux, voir Mayer et Laforest, $1990: 21$. Sur la définition du travail social, voir Laforest, 1984. Le terme "travail social » doit cependant être entendu ici dans une acception large, couvrant toutes les pratiques correspondant à la définition, et non seulement celles menées par des agentes et agents appartenant formellement à la Corporation professionnelle des travailleurs sociaux (corporation à titre réservé).

2. Chez Gramsci, le concept de " catharsis» rend compte de ce double mouvement d'identification émotive et de distanciation cognitive. II revient à un collègue du Département de travail social de I'Université du Québec à Montréal d'avoir récemment mis en valeur ce concept dans l'œuvre de Gramsci et d'en avoir dégagé la pertinence pour le travail social (Jouthe, 1990).

3. Pour toute la dynamique qui va de l'expérience fondatrice à l'institutionnalisation, en passant par le mouvement, je me suis librement inspiré de Alberoni, 1989. Depuis Statu nascenti (1968) jusqu'à Genesi (1989), en passant par Movimento e istituzione (1977 et 1981), Alberoni poursuit l'approfondissement d'une réflexion sur les mouvements sociaux et culturels à l'état naissant. Son concept clé est celui de "statu nascente », littéralement « état naissant », mais que j'ai traduit par « expérience fondatrice » ou « moment fondateur », pour qu'il ait plus de sens en français. L'idée de ce concept lui est venue à la lecture de l'allusion de Weber (1971 [1921] : 256) concernant le charisme "statu nascendi », expression latine qu'on pourrait traduire littéralement par « à l'état de ce qui est en train de naître ». Pour le concept d'identité, je me suis inspiré de Pizzorno, 1977, 1980, 1983a, 1983b. Pour le concept de marge, aucune influence précise n'a joué au départ, mais en cours de rédaction j'ai découvert Corin, 1986 par Guay, 1991a. Pour le concept de catharsis auquel il est fait allusion en début (note 2) et fin d'article, voir Jouthe, 1990.

4. Les autres documents qui ont préparé cette réforme sont: Pour un partenariat élargi : projet de politique de santé mentale pour le Québec (Harnois, 1987), Pour améliorer la santé et le bien-être au Québec : Orientations (MSSSQ, 1989a) et Politique de santé mentale (MSSSQ, 1989b).

5. Sur les " community councils » et " community chests », voir Médard, 1969 : 245-256.

6. En 1954, dans sa thèse de maîtrise en service social, le même abbé Riendeau justifiait le contrôle exercé par l'évêque sur les services sociaux diocésains : Riendeau, 1954 : 18.

7. Concernant ces expériences, voir : Médard, 1969 : 146-185.

8. Le titre de l'annexe 22, La voix des hommes sans voix, du Rapport de la Commission d'enquête sur la santé et le bien-être social (Castonguay et Nepveu, 1971) sera indirectement emprunté à l'abbé Pierre (1959 : 49), autre indice de I'influence de celui-ci au Québec.

9. Sur les développements de l'intervention du Conseil des œuvres de Montréal, dans le quartier Saint-Henri, avec I'arrivée de Michel Blondin, voir Blondin, 1965. 
10. Le développement communautaire a été conçu par les Anglais pour leurs colonies et ex-colonies et repris par l'Organisation des Nations Unies, dans les années 50 (ONU, 1955).

11. Le Bureau d'aménagement de l'est du Québec (BAEQ) a lui-même emprunté le terme " animation sociale » au mouvement français d'inspiration chrétienne sociale «Économie et humanisme», promoteur en France et en Afrique francophone d'une approche d'intervention qui intègre la participation des populations locales comme dimension fondamentale du développement. La figure intellectuelle dominante de ce mouvement est le père Louis-Joseph Lebret, auteur notamment de Suicide ou survie de l'Occident (1958). À noter ici que l'organisation communautaire naissante, marge de la profession du service social, croise de nouveau la marge progressiste de l'Église catholique. Il y a même ici recoupement d'influence avec celle précédemment évoquée de l'abbé Pierre. En effet, dans les années 50, une association appelée Association mondiale de lutte contre la faim et la misère était née $d^{\prime}$ une rencontre entre l'abbé Pierre, Josué de Castro, ex-directeur de la FAO et auteur de Géopolitique de la faim (1952), et le père Lebret (L'abbé Pierre, 1959 : 139). En 1976, les Éditions du Seuil publieront une traduction de Rules for Radicals (1971) d'Alinsky, sous le titre Manuel de l'animateur social. Cet intitulé est un syncrétisme terminologique de l'éditeur et du traducteur. II ne correspond pas aux termes de référence dans lesquels Alinsky définit lui-même sa méthode d'intervention.

12. L'évolution ultérieure de l'animation sociale au BAEQ et l'impasse où elle a abouti ont fait l'objet de plusieurs analyses, notamment celle de Simard (1978). La conjonction des marges de la technocratie et de l'organisation communautaire naissante aura cependant incité cette dernière à une mise en perspective du pôle de la participation en regard de celui de la rationalité exprimée dans la planification. Au contact du sociologue Gérald Fortin, consultant au BAEQ et professeur au Département de sociologie de l'Université Laval, où il s'inscrit en septembre 1965, Michel Blondin assume l'idée de cette complémentarité du « plan global » et de la participation comme deux éléments nécessaires de I'animation sociale (Blondin, 1965). Cette complémentarité ne trouvera guère I'occasion de s'actualiser cependant, puisque les politiciens et technocrates auxquels seront confrontés les " comités de citoyens » issus de l'animation sociale refuseront en général de se prêter aux exigences qu'elle comporte. Selon Fortin, la planification débouche elle-même sur un projet de société qu'il nomme " société de participation », mais qui est principalement axé sur le développement d'une participation consultative par l'État (Fortin, $1968 ; 53-60$ ). On trouvera une élaboration de ce projet de société dans l'annexe 25, La société de demain, ses impératifs, son organisation (Fortin, 1970), du Rapport de la Commission d'enquête sur la santé et le bien-être social (Castonguay et Nepveu, 1967-1972). Gérald Fortin, qui signe cette annexe, est alors « conseiller en développement social » auprès de la Commission.

13. Une attention à caractère sociologique et historique devrait être portée au croisement qui se produit ici entre une reviviscence du premier moment fondateur de l'organisation communautaire comme méthode participative de travail social en milieu anglo-saxon, avec les University Settlements, au XIXe siècle, et le moment fondateur de l'organisation communautaire dans le Québec francophone de la Révolution tranquille. Sur le mouvement des University Settlements, voir : Médard, 1969 : 232-245. 
14. Alberoni définit les mouvements culturels comme des mouvements " où sont protagonistes les intellectuels et les scientifiques »(Alberoni, 1989:15; je traduis).

15. Ce nombre sera ultérieurement porté à quatre sur quatorze (L.R.Q,. chap. S-5, à jour au 17 mars 1987 : art. 78).

16. Le manque de données historiographiques précises sur les acteurs et actrices, les circonstances et les rapports politico-institutionnels qui ont conduit à ce résultat doit être noté ici.

17. En 1985, le nombre de bénévoles était estimé à 900000 au Québec, soit $15 \%$ de la population (Laforest et Redjeb, $1989 ; 25)$. Ces bénévoles se répartissent également entre l'action directe et la gestion des organismes bénévoles (Malenfant, 1991 : 54-55).

18. Programme EXTRA («EXpérience de TRAvail ») : 43 \$ par mois ajoutés au chèque d'aide sociale pour 20 heures de travail par semaine, soit un salaire horaire de $0,54 \$$; en cas de refus, coupure de 109 \$ dans le chèque d'aide sociale. Programme STAGE (stage en milieu de travail) : 100 \$ par mois versés par l'employeur et s'ajoutant au chèque d'aide sociale pour 40 heures de travail par semaine, soit un salaire horaire de 0,62 \$. Étant de plus en plus admis aujourd'hui qu'un travail effectué dans un cadre bénévole puisse être rémunéré en deçà du « prix du marché » (Laforest et Redjeb : 35-36), ce n'est qu'en ce sens dérisoire qu'on peut assimiler au bénévolat les activités imposées aux personnes assistées sociales. Leur caractère obligatoire contredit fondamentalement " les caractères gracieux (au sens de gratuit), militant et libre » qui sont associés à la signification du bénévolat (Laforest et Redjeb : 35) ; d'où cette expression intentionnellement contradictoire de " bénévolat forcé ». Pour une interprétation favorable au programme EXTRA par un organisateur communautaire, voir : Archambault, 1992.

19. En soustrayant des 357 membres de la population de l'enquête de 1988 (AA.VV., 1989), les 33,1 \% qui exercent la fonction de gestion de second niveau décrite plus haut, on arrive au chiffre de 239 praticiens et praticiennes de l'organisation communautaire au sens spécifique, avec ou sans le titre, dans le réseau public, en $1988^{20}$. Pour ce qui est des groupes communautaires, le calcul est plus complexe. Un bottin publié par la Corporation de développement communautaire des Bois-Francs, en 1987, en dénombre 3500 (Corporation, 1987) en comptant les 158 CLSC qu'il faut ici exclure, soit une population de 3342 aux fins de nos calculs $^{21}$. Par ailleurs, une enquête par échantillon réalisée en 1989 par les Services aux collectivités de I'Université du Québec à Montréal (UQAM), avec la collaboration du Centre de formation populaire (Dumais et Côté, 1989), a porté sur 260 répondants et répondantes appartenant à 150 groupes communautaires $^{22}$, soit une moyenne de 1,7 personne salariée par groupe communautaire. En appliquant ce coefficient de 1,7 aux 3342 groupes de notre population, on arrive au total de 5681 personnes salariées dans les groupes communautaires. Par ailleurs, dans l'enquête citée, seulement 23,9\% des répondants et répondantes déclarent occuper une fonction correspondant à la catégorie " animation et intervention ». Les autres s'occupent de direction $(17,4 \%)$, de secrétariat $(4,6 \%)$, de financement $(1,5 \%)$, de comptabilité et tenue de livre $(2,3 \%)$, d'information et communication $(7,7 \%)$ et remplissent d'autres tâches $(6,6 \%)$. Par ailleurs, 35,9\% des répondants et répondantes entrent dans la catégorie «Deux postes et plus » (Dumais et Côté, 1989 : 34). Comme la combinaison de deux postes est fortement susceptible d'inclure un poste 
$d^{\prime}$ « animation et intervention », nous considérons, aux fins de notre extrapolation, que les deux catégories s'additionnent pour constituer le personnel total des intervenantes et intervenants sociaux dans les groupes communautaires, soit $59,8 \%(23,9 \%+35,9 \%)$ de l'ensemble. Appliqué à notre population de 5681 , ce coefficient nous amène à estimer ce personnel social à 3397 personnes. II reste maintenant à estimer la proportion d'entre elles qui exercent des fonctions $\mathrm{d}^{\prime}$ organisation communautaire au sens propre. Dans les CLSC, cette proportion est de $12,2 \%{ }^{23}$. En supposant que ce dernier coefficient soit applicable à notre estimation du personnel social dans les groupes communautaires, on arrive au total hypothétique, mais plausible, de 414 personnes exerçant des fonctions d'organisation communautaire au sens propre, dans les groupes communautaires. En additionnant ce résultat à celui obtenu dans les CLSC (239), on obtient le total probable de 653 salariés ou salariées exerçant à temps plein ou à temps partiel le métier d'organisateur ou d'organisatrice communautaire au sens propre, avec ou sans le titre, en 1988-1989, au Québec.

20. Entre l'enquête du ROCQ effectuée en 1978 (Doré et Larose, 1979) et celle de 1988 (AA.VV., 1989), tous les postes d'organisation communautaire du réseau public ont été transférés vers les CLSC. Dans l'enquête de 1988, un des modèles de pratique auxquels se rattachent $11,3 \%$ des répondants et répondantes nous laisse perplexe pour ce qui est de sa correspondance à la spécificité de I'organisation communautaire, en ce qui a trait à la participation des personnes elles-mêmes aux prises avec les problèmes et au changement social, en regard de celle du service social des groupes. II s'agit de l'animation de groupe « qui permet à un groupe de clarifier ses orientations, d'identifier les problèmes à résoudre, les ressources dont il dispose et le solutions éventuelles ; I'intervenant/e a comme mandat de faciliter l'atteinte des objectifs que le groupe s'est fixés » (AA.VV., 1989 : 83). Aux fins de la présente extrapolation, nous laissons la question ouverte et comptons les adhérents et adhérentes à ce modèle parmi les tenants et tenantes de l'organisation communautaire comme pratique sociale spécifique.

21. Parmi ces organismes communautaires, 1500 sont financés par le ministère de la Santé et des Services sociaux (David, 1991 : 45).

22. L'information relative au nombre de groupes auxquels appartenaient les répondants et répondantes n'est pas contenue dans le rapport de l'enquête (Dumais et Côté, 1989). Elle m'a été fournie par Josée Belleau du Centre de formation populaire, le 21 novembre 1989.

23. Cette proportion est obtenue en mettant en rapport le nombre de travailleurs et travailleuses communautaires $(96,1)$, travailleurs et travailleuses de quartier $(23,4)$ et organisateurs et organisatrices communautaires $(225,8)$, diminué de $33,1 \%$ (pour tenir compte de ceux et celles d'entre eux et elles affectés à I'« organisation de services» (AA.VV., $1989: 13$ et 83), avec le nombre total d'intervenantes et intervenants sociaux dans les CLSC, en comprenant sous ce terme les travailleuses et travailleurs sociaux professionnels, techniciennes et techniciens en assistance sociale, psychologues, travailleuses et travailleurs communautaires, travailleuses et travailleurs de quartier, organisatrices et organisateurs communautaires, agentes et agents de relations humaines et autre personnel en travail social (sans inclure les auxiliaires familiales) qui forment ensemble un total de 1891,3 . L'opération est la suivante : 96, $1+23,4+225,8$ $=345,3-33,1 \%=231$ divisé par $1891,3=12,2 \%$. La source des données est MSSSQ, 1990. Il s'agit de la situation au 31 mars 1989 et les données sont 
fournies en équivalent plein temps, d'où les fractions décimales affectées à chaque catégorie.

24. Statistiques compilées à partir du fichier des membres, 1989-1990 et 1990-1991.

25. Tiens-toé : qui deviendra Inter-action, puis Inter-action communautaire.

26. Informations transmises à l'Assemblée générale du 26 mai 1990 du RQIIAC.

27. Dans le même paragraphe, on peut lire en caractères ordinaires : «...l'action communautaire constitue un mode d'intervention approprié pour mobiliser les populations et les renforcer dans leur volonté d'apporter des solutions collectives aux problèmes socio-sanitaires qui les affligent »... et plus loin, en caractères gras cette fois : " Le CLSC entreprendra les actions communautaires requises pour susciter une mobilisation des responsables et des forces vives du milieu qui aideront les personnes, surtout celles qui sont plus vulnérables, à mieux fonctionner dans leur quotidien » (FCLSC, 1991 : 14).

28. Sur la catharsis comprise dans le sens évoqué ici, voir : Jouthe, 1990.

29. Métahistorique : au sens où elle transcende telles circonstances historiques particulières.

\section{Références bibliographiques}

AA. VV. (1989). Pratiques d'organisation et de travail communautaires en CLSC. Montréal : Regroupement québécois des intervenants et intervenantes en action communautaire en CLSC (C.P. 43, succ. M, Montréal, H1V 3L6).

AA. VV. (1987). Fais-moi signe de changement. Les actes du colloque provincial sur le développement communautaire. Victoriaville : Corporation de développement communautaire des Bois-Francs inc.

AA. VV. (1985). Profil socio-économique des coopératives de travail au Québec. Montréal : Université du Québec à Montréal.

AlARY, J. (dir.) (1988). Solidarités. Pratiques de recherche-action et de prise en charge par le milieu. Montréal : Boréal.

AlBERONI, F. (1968). Statu nascenti. Bologna : Il Mulino.

AlBERONI, F. (1977 et 1981). Movimento e istituzione. Teoria generale. Bologna : II Mulino.

AlBeroni, F. (1989). Genesi. Milano : Garzanti. Citations traduites par Gérald Doré.

ALINSKY, S.D. (1969 [1946]). Reveille for Radicals. New York : Random.

ALINSKY, S.D. (1971). Rules for Radicals. New York: Random. Ce livre a été traduit en français sous le titre Manuel de l'animateur social. Paris : Seuil, 1976.

AMPLEMAN, G. (1987). "La formation des intervenantes en santé communautaire en milieux populaires », dans AA. VV., Pratiques de conscientisation 2, Québec : Collectif québécois d'édition populaire (C.P. 428, Saint-Sauveur, Québec [Québec], G1K 6W8) : 179-213.

Archambault, R. (1992). "Les programmes EXTRA : Qui exploite qui ? », Inter-action communautaire, vol. $\mathrm{V}, \mathrm{n}^{\circ} 4: 9-11$. 
AUCLAIR, R. et R. BÉLAND (1983). "Chômeurs et chômeuses : le Mouvement ActionChômage (MAC) de Portneuf ", Service social, vol. 32, n 3 : 385-401.

BAEQ (1966a). "Participation », dans Plan de développement, chap. l, cahier 1. Sans lieu : BAEQ : 27-33 et 38-39.

BAEQ (1966b). "Politique d'animation », dans Plan de développement, chap. V, cahier 8. Sans lieu : BAEQ. : 132-143.

BARCLAY, P.M. (ed.) (1982). Social Workers : Their Role \& Tasks. London : Bedford Square Press.

BEAUGRAND-CHAMPAGNE, G. (1968). Quelques réflexions sur les rapports entre l'éducation des adultes et le développement communautaire. Communication lors de la journée d'étude sur "L'animation au Québec », 2 mars. Québec, Université Laval : Faculté des sciences sociales.

BLONDIN, M. (1965). "L'animation sociale en milieu urbain : une solution », Recherches sociographiques, vol. VI, no $3: 283-304$.

BLONDIN, M. (1970). "Entrevue », dans F. Lesemann et M Thiénot (dir.) (1972) Animations sociales au Québec. Rapport de recherche. Université de Montréal, École de service social : 299-317.

BLONDIN, M. (1987). « Animation en milieu ouvrier : de Saint-Henri à la FTQ », dans P.R. Bélanger, B. Lévesque, R. Mathieu et F. Midy (dir.), Animation et culture en mouvement. Sillery : Presses de I'Université du Québec : 25-32.

BLONDIN, M. (1989 [1966]). "Service social et pauvreté en milieu urbain », Nouvelles pratiques sociales, vol. 2, $\mathrm{n}^{\circ} 1: 113-117$.

BOIVIN, R. (1988). Histoire de la Clinique des citoyens de Saint-Jacques (1968-1988). Montréal : VLB.

BOUCHER, P.-A. (1982). Tricofil, tel que vécu. Montréal : Éditions CIRIEC.

BOURQUE, D. (1989). L'approche communautaire et l'action communautaire : plus $q u$ 'un mariage de raison. Montréal : Regroupement québécois des intervenants et intervenantes en action communautaire en CLSC (C.P. 43, succ. M, Montréal [Québec], H1V 3L6).

Castonguay, C. et G. NePVeu (dir.) (1967-1972). Rapport de la Commission d'enquête sur la santé et le bien-être social. Québec : Gouvernement du Québec.

CENTRE COOPÉRATIF DE RECHERCHE EN POLITIQUE SOCIALE (1975). Les coopératives ouvrières de production et la lutte des travailleurs. Sainte-Foy : CEQ.

COCQ (1988). Pour la reconnaissance de l'action communautaire autonome. Montréal : Coalition des organismes communautaires du Québec.

COMEAU, Y. (1989). "Le réseau de la conscientisation au Québec », Possibles, vol. $13, \mathrm{n}^{\circ} 4: 131-140$.

CORPORATION DE DÉVELOPPEMENT COMMUNAUTAIRE DES BOIS-FRANCS INC. (1987). Le bottin communautaire du Québec. Victoriaville : Corporation de développement communautaire des Bois-Francs inc.

CQC (1983). Manifeste. Québec : Collectif québécois de conscientisation (C.P. 25, Limoilou, Québec [Québec], G1L 4T8).

CORIN, E. (1986). "Centralité des marges et dynamique des centres », Anthropologie et sociétés, vol. 10, $\mathrm{n}^{\circ} 2: 1-21$.

DANIEL, C. (1987). "Des précisions sur la notion de communautaire », dans AA. VV., Fais-moi signe de changement. Les actes du colloque provincial sur le développement communautaire. Victoriaville : Corporation de développement communautaire des Bois-Francs inc. 
DAVID, F. (1991). La réforme Lavoie-Roux-Côté : entre la vertu et la pratique... tout un océan d'incertitudes. Montréal : L'R des centres de femmes du Québec et le Regroupement des ressources alternatives en santé mentale du Québec.

DE CASTRO, J. (1952). Géopolitique de la faim. Paris : Éditions ouvrières.

DESLAURIERS, J.-P. ET H. POULIOT (1982). Les groupes populaires à Sherbrooke : pratique, financement et structure. Université de Sherbrooke : Département de service social.

DIONNE, H. (1979). «Introduction : animation sociale et développement régional du BAEQ à nos jours ", dans B. Lévesque (dir.), Animation sociale, entreprises communautaires et coopératives, Montréal : Saint-Martin, p. 49-54.

DORAY, M. (1967). " Méthodes et techniques d'animation », Les cahiers de I'I.C.E.A., $n^{o s} 4-5: 23-37$.

DORÉ, G. (1978). La politique sociale comme pratique de classes. Thèse de doctorat en sociologie. Québec : Université Laval.

DORÉ, G. (1985). «L'organisation communautaire : définition et paradigme », Service social, vol. $34, \mathrm{n}^{\text {os }} 2-3: 210-230$.

DORÉ, G. (1991). "L'organisation communautaire et l'éthique de la solidarité », Service social, vol. 40, no $1: 125-141$.

DORÉ, G. et C. LAROSE, (1979). "L'organisation communautaire : pratique salariée d'animation des collectivités au Québec », Service social, vol. 28, $\mathrm{n}^{\text {os }} 2-3$, $1979: 69-96$.

DUMAIS, S. et R. CÔTÉ (1989). Enquête sur les conditions de salaire et les conditions de travail des travailleuses et travailleurs au sein des groupes populaires. Montréal : Service aux collectivités de l'Université du Québec à Montréal.

FAS (CSN) (1990-1991). Convention collective intervenue entre le Comité patronal de négociation du secteur de la santé et des services sociaux, le sous-comité patronal de négociation des centres locaux de services communautaires et la Fédération des Affaires sociales (CSN). Montréal : Fédération des affaires sociales de la Confédération des syndicats nationaux. La description du titre d'emploi « organisateur ou organisatrice communautaire » est la même depuis la signature de la première convention collective.

FAVREAU, L. (1989). Mouvement populaire et intervention communautaire de 1960 à nos jours. Montréal : Le Centre de formation populaire.

FCLSC (1991). Groupe de travail sur l'action communautaire en CLSC. Montréal : Fédération des centres locaux de services communautaires.

FORTIN, D. et M. ROLAND (1980). Histoire des luttes de protection des consommateurs 1962-1978. Québec: Université Laval, École de service social, Groupe de recherche en action populaire (GRAP).

FORTIN, G. (1968). Participation et société. Québec : Université Laval, Département de sociologie.

FORTIN, G. (1970). La société de demain : ses impératifs, son organisation. Annexe 25. Gouvernement du Québec : Commission d'enquête sur la santé et le bienêtre social.

FOURNIER, J. (1991). "Les groupes communautaires et les CLSC : des rapports de porcs-épics », Inter-action communautaire, vol. V, $\mathrm{n}^{\circ} 3: 10-13$.

GAUDREAU, L. (1983). «Assistés sociaux et assistées sociales : I'Association pour la défense des droits sociaux du Québec métropolitain (ADDSQM) ", Service social, vol. 32, nº $3: 355-381$. 
GINGRAS, P. (1988). L'approche communautaire : essai de conceptualisation. Québec : Université Laval, Centre de recherche sur les services communautaires.

GIRARD, C. (1989). "Le Mouvement action-chômage de Montréal : 20 ans de pratique sociale", Nouvelles pratiques sociales, vol. 2, $\mathrm{n}^{\circ} 2: 37-48$.

GMATI, A. et J. ROBITAILLE, J. (1991). «L'aide sociale et l'« employabilité » : cheap labor au programme », $V O, \mathrm{n}^{\circ} 233: 26-38$.

GRAND'MAISON, J. (1967). "L'action de la base », Socialisme '67, no 11 : 79-98.

GUAY, J. (1991a [1989]). "Ce que quelques organismes communautaires en pensent... ", Inter-action communautaire, vol. V, $\mathrm{n}^{\circ} 1: 8$.

GuAY, L. (1991b). Le choc des cultures : bilan de l'expérience de participation des ressources alternatives à l'élaboration des plans régionaux d'organisation de services en santé mentale. Montréal : Regroupement des ressources alternatives en santé mentale du Québec (RRASMQ).

HARNOIS, G.P. (dir.) (1987). Pour un partenariat élargi : projet de politique de santé mentale pour le Québec. Québec : Gouvernement du Québec, ministère de la Santé et des Services sociaux, Comité de la politique de santé mentale.

HOULE, G. (1972). «L'animation sociale en milieu urbain : une idéologie pédagogique », Recherches sociographiques, vol. XIII, nº $2: 231-253$.

HURTUBISE, Y. (1991). "L'action conscientisante », dans L. Doucet et L. Favreau (dir.). Théorie et pratiques en organisation communautaire, Sillery : Presses de I'Université du Québec : 147-159.

HuRTUBISE, Y. et P. PARÉ (dir.) (1989). Pratiques d'action communautaire en CLSC. Actes du colloque sur I'action communautaire en CLSC, tenu à I'Université Laval (Québec) les 12, 13 et 14 mai 1988. Québec : Université Laval, Centre de recherches sur les services communautaires.

JAL (LES TRAVAILLEURS DU) (1979). "Le JAL », dans B. Lévesque (dir.), Animation sociale, entreprises communautaires et coopératives. Montréal : Saint-Martin.

JOUTHE, E. (1990). Catharsis et transformation sociale dans la théorie politique de Gramsci. Sillery : Presses de I'Université du Québec.

L'ABbÉ PIERRE (1959). L'abbé Pierre parle aux Canadiens et aux heureux du monde entier. Montréal : Les éditions de l'Homme.

LABRIE, V. (1985). "Garderies populaires : la maternité devant l'autorité », Autogestions, $\mathrm{n}^{\text {os }} 20-21: 71-76$.

LACHAPELLE, R. (1990). L'approche communautaire en CLSC. Un concept révélateur. Sorel : Centre local de services communautaires du Havre.

LAFOREST, J. (1984). "La nature du service social (la compétence des travailleurs sociaux) », Intervention, $\mathrm{n}^{\circ} 68: 22-32$.

LAFOREST, J. (1983). «Les personnes âgées et les inégalités socio-économiques : I'Association québécoise pour la défense des droits des retraités et préretraités (AQDR) », Service social, vol. 32, no 3 : 433-448.

LAFOREST, M. et B. REDJEB (1989). L'intervention sociale non salariée : étude exploratoire de dix pratiques d'assistance bénévole auprès des personnes âgées dans la région de Québec. Québec : Université Laval, École de service social.

LALONDE-GRATON, M. (1985). La p'tite histoire des garderies. Saint-Lambert : Regroupement des garderies de la région 06-C.

LAMOUREUX, J. (1988). "La commission Rochon : le communautaire, encore et toujours à la marge », Revue internationale d'action communautaire, 20/26 : 157-170. 
LAPLANTE, P. (1962). "L'organisation communautaire telle qu'on la pratique au Conseil des œuvres de Montréal », Service social, vol. 11, nº 1 : 110-115.

LAROCHELLE, G. et S. ROBICHAUD (1991). "Contestation et attestation étatique : le nouveau catéchisme des groupes communautaires au Québec », Revue française de science politique, vol. 41, $\mathrm{n}^{\circ} 5$ : 649-675.

LAROSE, C. (1983). "Prendre le droit de parole », dans AA. VV., Pratiques de conscientisation, Montréal : Nouvelle Optique : 131-153.

LEBEL, J.-C. (1967). "L'animation sociale : expérience du BAEQ », Les Cahiers de I'ICEA, $\mathrm{n}^{\circ} 3: 57-75$.

LEBRET, L.-J. (1958). Suicide ou survie de l'Occident ? Paris : Éd. Ouvrières.

LE SOLEIL (1991). "L'Archipel d'Entraide est au bord du gouffre », Le Soleil, jeudi 28 novembre : B-16.

LÉVESQUE, B. (dir.) (1979). Animation sociale, entreprises communautaires et coopératives. Montréal : Saint-Martin.

MALENFANT, R. (1991). "Le bénévolat : un domaine inexploré de la recherche », dans AA. VV., Les liens entre la recherche et l'intervention en service social : problématique et orientation, Québec, Université Laval : École de service social, p. 51-65.

MARTIN, C. (1990). "Compte rendu des ateliers de travail : pour mieux connaître et comprendre les milieux populaires», dans AA. VV., Actes du forum "Les inégalités socio-économiques et la santé ». Québec : Gouvernement du Québec, ministère de la Santé et des Services sociaux : 37-41.

MAS (1971). Les centres locaux de services communautaires (CLSC). Québec : ministère des Affaires sociales.

MAYER, R. et M. LAFOREST (1990). "Problème social : le concept et les principales écoles théoriques", Service social, vol. 39, n 2 : 13-43.

MCGRAW, D. (1978). Le développement des groupes populaires à Montréal (19631973). Montréal : Saint-Martin.

MÉDARD, J.-F. (1969). Communauté locale et organisation communautaire aux ÉtatsUnis. Paris : Armand Colin.

MEPACQ (1984). "Si vous aimez les « petites histoires » : Déjà 20 ans ! » Supplément au journal L'ardoise, vol. 2, n 3 : III-VII.

MORENCY, M.-A. (1966). Animation sociale : quelques éléments historiques de l'expérience du BAEQ. Annexe technique du plan de développement, $\mathrm{n}^{\circ} 20$. Sans lieu : BAEQ.

MORIN, C. (1962). "La méthode d'organisation communautaire dans le milieu canadien-français », Service social, vol. 11, n $1: 46-63$.

MSSSQ (1989a). Pour améliorer la santé et le bien-être au Québec : Orientations. Québec : Gouvernement du Québec, ministère de la Santé et des Services sociaux.

MSSSQ (1989b). Politique de santé mentale. Québec : Gouvernement du Québec, ministère de la Santé et des Services sociaux.

MSSSQ (1990). État de situation des CLSC et des centres de santé. Données régionales et provinciales au 31 mars 1989. Québec : Gouvernement du Québec, ministère de la Santé et des Services sociaux, Direction générale de la prévention et des services communautaires.

ONU (1955). Social Progress through Community Development. New York : United Nations. 
PANET-RAYMOND, J. (1985). "Nouvelles pratiques des organisations populaires... Du militantisme au bénévolat au service de l'État », Service social, vol. 34, $\mathrm{n}^{\text {os }} 2-3: 340-352$.

PARAZELLI, M. (1988). " L'action communautaire autonome mise en tutelle! », Interaction, vol. II, $\mathrm{n}^{\circ} 4: 1-4$.

PARAZELLI, M. (1990). "Pour ajouter de la misère à la vie. L'impact d'une épidémiologie socio-étatique sur l'action communautaire et les problèmes sociaux ", Service social, vol. 39, $\mathrm{n}^{\circ} 2$ : 175-187.

Pelletier, D. (1973). " "SEAPAC " et les centres communautaires », ÉducationQuébec, vol. 3, n $10: 16-19$.

PERRON, J. (1984). "L'évolution des services sociaux d'expression française au Québec entre 1940 et 1960 », Intervention, nº 69 : 17-27.

PizzORNO, A. (1977). "Scambio politico e identità collettiva nel conflitto di classe », Revista italiana di scienza politica, a. VII, $\mathrm{n}^{\circ} 2$ : 165-198.

Pizzorno, A. (1980). I soggetti del pluralismo. Classi. Partiti. Sindacati. Bologna : II Mulino.

PizzornO, A. (1983a). "Sulla razionalità della scelta democratica », Stato e mercato, $\mathrm{n}^{\circ} 7: 3-46$.

Pizzorno, A. (1983b). "Identità e interesse », in Sciolla, L. (a cura di), Identità : percorsi di analisi in sociologia. Torino : Rosenberg e Sellier.

POIRIER, R. (1986). Qui a volé la rue principale ? Montréal : Éditions Départ.

PRUD'HOMmE, R. (1961). Rapport d'activités. Re : Projet-Conseil de quartier. Montréal : Conseil des œuvres.

QUÉBEC (1971). Loi sur les services de santé et les services sociaux, sanctionnée le 24 décembre 1971. Québec : Éditeur officiel du Québec.

QUÉBEC (1987). Loi sur les services de santé et les services sociaux, dans L.R.Q., chap. S-5, à jour au 17 mars 1987.

QUÉBEC (1991). Loi sur les services de santé et les services sociaux et modifiant diverses dispositions législatives. Québec : Éditeur officiel du Québec.

RENÉ, J.-F. et J. PANET-RAYMOND (1984). Faut-il brûler le pancartes ? Montréal : ACEF du centre de Montréal.

RIENDEAU, R. (1954). Nos services sociaux diocésains. Thèse de maîtrise en service social. Université de Montréal : École de service social.

ROCHON, J. (dir.) (1988). Rapport de la Commission d'enquête sur les services de santé et les services sociaux. Québec : Les Publications du Québec.

ROCQ (1979 [1977] ). Manifeste. Québec : Regroupement des organisateurs communautaires du Québec (C.P. 25, Limoilou, Québec [Québec], G1L 4T8).

ROCQ-CQC (1987). 1977-1987 : 10 ans déjà ! Québec : Collectif québécois de conscientisation (C.P. 25, Limoilou, Québec [Québec], G1L 4T8).

Rosanvallon, P. (1981). La crise de l'État-Providence. Paris : Seuil.

Ross, M.G. (1955). Community Organization : Theory and Principles. New York : Harper.

RQIIAC (1991). Colloque biennal. L'action communautaire en CLSC : des acquis, des défis. Programme préliminaire. Montréal : Regroupement québécois des intervenants et intervenantes en action communautaire en CLSC (C.P. 43, succ. M., Montréal [Québec], H1V 3L6). 
SIMARD, J.-J. (1979). «De l'utopie à I'idéologie : planification, participation et pouvoir technocratique », dans B. Lévesque (dir.), Animation sociale, entreprises communautaires et coopératives. Montréal : Saint-Martin : 299-317.

THÉÂTRE PARMINOU (1987). "Le théâtre d'intervention, une alternative culturelle », dans P.R. Bélanger, B. Lévesque, R. Mathieu et F. Midy (dir.), Animation et culture en mouvement. Sillery : Presses de l'Université du Québec : 181-188.

THÉÂTRE SANS DÉTOUR (1982). «Opprimés, exprimez-vous sans détour ! », Luttes urbaines, vol. $3, \mathrm{n}^{\circ} 2: 38-41$.

SOULIÉ, J.-P. (1991). "Des CLSC exploitent les assistés sociaux », La Presse, no 25 : A-1 et A-2.

UNIVERSITÉ LAVAL (1991-1992). «SVS-63082 Séminaire sur Paulo Freire » et « SVS63084 Mouvements populaires II 》, dans Répertoire des cours des deuxième et troisième cycles. Québec : Université Laval, p. 135-136.

VALLÉE, L. (1991). « Le document de réflexion de la Fédération des CLSC du Québec. L'Action communautaire enfin reconnue ! ", Inter-action communautaire, vol. $\mathrm{V}, \mathrm{n}^{\circ} 2: 1-3$.

VALLIÈRES, C. (1985). "Combien de cordes à mon A.R.C.Q. ? ", Autogestions, nos 20$21: 189-198$.

VeNTELOU, D. (1983). "Le point de départ : une lutte », dans AA. VV., Pratiques de conscientisation. Montréal : Nouvelle Optique, p. 15-39.

WeBER, M. (1971 [1921] ). Économie et société, tome I. Paris : Plon. 